\title{
1 Molecular Epidemiology of African Sleeping Sickness
}

2

3 GEOFF HIDE ${ }^{\mathrm{a}}$ AND ANDREW TAIT

4

$5 \quad{ }^{a}$ Centre for Parasitology and Disease, Biomedical Sciences Research Institute, School of

6 Environment and Life Sciences, University of Salford, Salford, UK, M5 4WT

7

$8{ }^{b}$ Faculty of Veterinary Medicine, Division of Infection and Immunity, University of Glasgow,

9 Bearsden Road, Glasgow, G61 1QH, Scotland, U.K.

11 Corresponding Author:

12 Geoff Hide

13 Centre for Parasitology and Disease, Biomedical Sciences Research Institute, School of

14 Environment and Life Sciences, University of Salford, Salford, UK, M5 4WT

15 Telephone No:

01612953371

16 Fascimile No:

01612955015

17 E-mail No:

g.hide@salford.ac.uk

20 Running title: Molecular epidemiology of African sleeping sickness

22 Keywords: African trypanosomiasis; Trypanosoma brucei; sleeping sickness; molecular 23 epidemiology; epidemics; microsatellites; genetics; population structure 


\section{SUMMARY}

2 Human sleeping sickness in Africa, caused by Trypanosoma brucei spp. raises a number of

3 questions. Despite the widespread distribution of the tsetse vectors and animal

4 trypanosomiasis, human disease is only found in discrete foci which periodically give rise to

5 epidemics followed by periods of endemicity A key to unravelling this puzzle is a detailed

6 knowledge of the etiological agents responsible for different patterns of disease - knowledge

7 that is difficult to achieve using traditional microscopy. The science of molecular

8 epidemiology has developed a range of tools which have enabled us to accurately identify

9 taxonomic groups at all levels (species, subspecies, populations, strains and isolates). Using

10 these tools, we can now investigate the genetic interactions within and between populations

11 of Trypanosoma brucei and gain an understanding of the distinction between human- and

12 nonhuman-infective sub species. In this review, we discuss the development of these tools,

13 their advantages and disadvantages and describe how they have been used to understand

14 parasite genetic diversity, the origin of epidemics, the role of reservoir hosts and the

15 population structure. Using the specific case of T.b.rhodesiense in Uganda, we illustrate how

16 molecular epidemiology has enabled us to construct a more detailed understanding of the

17 origins, generation and dynamics of sleeping sickness epidemics. 
3 Human sleeping sickness in Africa is a puzzling disease. It is caused by the parasite

4 Trypanosoma brucei (see table 1). Despite the widespread distribution of the tsetse vectors

5 and animal trypanosomiasis, human disease is found in very specific foci and appears as

6 epidemics followed by periods of endemicity (reviewed in Hide, 1999). The locations of these

7 foci are very stable over time but occasionally new epidemics emerge in new localities. For

8 example, the Busoga focus of Uganda has had epidemics recorded from 1900 to the present

9 day while a new epidemic has emerged in the nearby Soroti region - an area free of sleeping sickness prior to 1998 (Welburn et al., 2001). An understanding of the factors which cause

11 these epidemics could clearly lead to an understanding of how to eradicate the disease.

12 Sleeping sickness was one of the earliest examples of the application of molecular

13 epidemiology to a parasitic disease and, as will be seen, some important insights have been

14 made into the origins and dynamics of these epidemics. In this review, we will trace the

15 development of molecular epidemiological approaches with a particular emphasis on African

16 sleeping sickness. As the journal "Parasitology" has played a significant role in publishing

17 these developments it is an appropriate topic for the centenary issue.

\section{MOLECULAR EPIDEMIOLOGY}

The molecular epidemiology of parasites describes the application of DNA based techniques

21 to the understanding of parasite epidemiology and diversity (Hide \& Tait, 1991). The approach was prompted by an emerging understanding that parasites could not be easily packaged into groups of organisms that produced defined diseases in defined hosts. Instead, there were complex epidemiological cycles involving multiple hosts, sometimes multiple 
1 diseases, reservoir hosts, specific strains, emerging drug resistance, genetic exchange

2 between strains and even the apparent emergence of new parasite species. Thus tools were

3 needed which could be used to gain a higher level of resolution than morphology in defining

4 the organisms involved. In fact, the discipline of molecular epidemiology strictly emerged

5 from several earlier strands of research. Principally this was the use of phenotypic

6 approaches such as isoenzyme (or allozyme) analysis as a tool to study the epidemiology of

7 parasitic infections. One of the key epidemiological questions in parasitology is determining

8 the correct identity (diagnosis) and taxomomic level of a given parasite (eg. species,

9 subspecies, strains, variants). This has often proved challenging especially in the case of microscopic parasites (e.g. parasitic protozoa) where morphological distinctions are not

11 easily identified. Two linked problems exist - defining the scope of a taxonomic unit and establishing unambiguous markers for each unit. The scope of a taxonomic unit could be relatively easily established in the absence of any form of gene exchange between units such

14 as occurs in clearly distinct species or clonally reproducing parasites. However in earlier studies the precise identity of parasitic species or the role of gene exchange was often not known. An inability to clearly define taxonomic units therefore makes it difficult to identify markers that can be used diagnostically to distinguish that taxonomic unit. There is unfortunately a degree of circularity in the problem. There are two levels of identification needed: (1) diagnosis - generally referring to diagnostic tools capable of discriminating to the species level and defining a particular association between causative agent and disease and (2) epidemiological tools - generally referring to markers that can be used at the sub-species or strain level to investigate detailed interactions within parasite transmission cycles. This

23 problem of circularity in developing appropriate markers can be overcome by using independent markers that are not linked to the, often very subjective, medical criteria by 
1 which parasites were traditionally classified. DNA based molecular methods offered a

2 potential solution to these problems.

4 FROM BIOCHEMICAL TO MOLECULAR EPIDEMIOLOGY

6 The advent of protein based technologies such as gel electrophoretic separation of

7 isoenzymes (allozymes) enabled distinction of morphologically identical organisms and could

8 be used as a tool for genetic studies. These approaches were initially used with the free-

9 living protozoa both as markers for studying genetic exchange and for defining species

10 (previously defined as syngens) as illustrated by work with Paramecium aurelia (Tait, 1970a;

11 Tait, 1970b). Key centres in Edinburgh, London and subsequently Bristol then applied these

12 approaches to investigating the genetics and taxonomy of the medically important protozoa,

13 Plasmodium and Trypanosoma. For example these approaches were used for identifying

14 different levels of taxonomic units (eg species, subspecies and strains) in Plasmodium

15 (Carter \& Walliker, 1975) and Trypanosoma spp (Kilgour and Godfrey, 1973; Miles et al.,

16 1977; Godfrey ,1978; Gibson et al., 1978; Gibson et al., 1980; Tait et al., 1984, 1985).

17 Furthermore, using these techniques, genetic exchange was shown to occur in Plasmodium

18 spp (Walliker et al., 1971, 1973) and Trypanosoma spp (Tait,1980; Gibson et al., 1980; Jenni

19 et al., 1986; Gibson et al., 1989).

20 When used to analyse the African trypanosomes causing human sleeping sickness, these

21 approaches revealed a complex taxonomic situation. The classical taxonomy described three

22 subspecies, Trypanosoma brucei brucei, (non-human infective) Trypanosoma brucei

23 rhodesiense (East African, human infective) and Trypanosoma brucei gambiense (West

24 African, human infective). Multilocus enzyme electrophoresis (MLEE) studies revealed that 
1 there were clearly subgroups or strains within both T.b.gambiense and T.b.rhodesiense as

2 well as a high degree of variation between isolates of T.b.brucei (Gibson et al., 1980; Tait et

3 al., 1984, 1985). Furthermore, these studies showed that different levels of taxonomy

4 appeared to be operating - the genetic relationships showed that T.b.gambiense appeared to

5 behave like a separate subspecies while T.b.rhodesiense appeared to be a host range

6 variant of T.b.brucei.

7 It was becoming apparent that the question of what defines a species in parasitic protozoa

8 (and other micro-pathogens) was a complex one to address. Traditionally, the species

9 concept is based on presence or absence of genetic exchange between taxonomic units. In the case of T.brucei sspp, the extent of the occurrence of genetic exchange in natural

11 populations was unclear. Tibayrenc et al., 1990 conducted a reanalysis of MLEE data from a 12 wide range of pathogens including T.brucei and Plasmodium and proposed a clonal theory,

13 based on a range of measures such as high levels of heterozygosity, linkage disequilibrium 14 and over representation of particular genotypes. In the case of T.b.rhodesiense there was 15 evidence for clonality but the results with T.b.brucei were less convincing. Further analysis of 16 a large collection of isolates from across Africa (Mathieu-Daude \& Tibayrenc, 1994) was 17 interpreted as showing limited genetic exchange although geographical sub-structuring could 18 be an explanation given the diversity of the origins of the strains. These conclusions conflicted with published data showing the existence of genetic exchange (eg Tait and Turner, 1990). Analysis of a large collection of isolates from the Lambwe Valley focus, in 21 kenya, provided a partial resolution of the debate by showing that the population had an 22 epidemic population structure (Maynard-Smith et al., 1993) i.e. that is there was underlying 23 genetic exchange masked by the local expansion of a small number of genotypes. Further 24 analysis of populations from Cote d'Ivoire, Zambia and Busoga, Uganda, (Stevens and 
1 Tibayrenc, 1995) showed a diversity of population structures with evidence for clonality in

2 some populations but an epidemic structure in others (Uganda). This diversity was also

3 illustrated by MLEE analysis of sympatric stocks collected during a sleeping sickness

4 epidemic in Tororo, Uganda (Figure 1). In this study, it was demonstrated that the population

5 structure of the T.b.brucei stocks appeared to conform to a random mating population

6 structure while the population structure of T.b.rhodesiense was recognized as epidemic (Hide

7 et al., 1994). The debate over the role of genetic exchange in T.brucei was not fully resolved

8 using MLEE.

9 MLEE is a highly informative technique and enabled epidemiological analyses to be based on

10 a genetic interpretation, with the essential conclusions for African trypanosomes remaining

11 unchallenged today. However, these methods require relatively large quantities of pure

12 parasite material and so are cumbersome for large scale epidemiological analysis. Initially,

13 blood from patients and animals had to be isolated, followed by several passages through

14 mice (or in culture) and further mouse amplification to produce enough material for analysis.

15 In some cases, the Kit for In Vitro Isolation (KIVI) of trypanosomes has been used to isolate

16 trypanosomes (Truc et al., 1992). During these processes, cloning from individual

17 trypanosomes was also often necessary with several years work required to analyse an

18 appropriately sized sample set. An additional problem associated with these methods is the

19 potential of the methods to select for particular types of trypanosomes, such as those better

20 adapted to growth in mice, and thereby introduce bias into the distribution of parasites

21 recovered (Koffi et al., 2007).

22 Initially, DNA technology was focussed on developing diagnostic tools to identify

23 Trypanosoma species. As it is the subject of another review in this issue (Gibson, 2009), it

24 will not be considered further here and we will consider the development of DNA based tools 
1 for measuring intraspecific variation (Table 2). Initial DNA studies on intraspecific variation in

2 T.brucei were based on analysis of restriction enzyme site variation in the kinetoplast DNA

3 (Borst et al., 1981). However with limited stocks available and a relatively small number of

4 restriction enzyme sites covered, it was not possible to discriminate between the three

5 subspecies. A larger scale analysis (Gibson et al., 1985) however demonstrated that a

6 difference could be detected in stocks originating from East and West Africa. Restriction

7 Fragment Length Polymorphism (RFLP) of VSG antigen genes and numerical taxonomy,

8 based on those RFLPs, revealed that T.b.gambiense was quite distinct from T.b.rhodesiense

9 or T.b.brucei but that the latter two subspecies could not be distinguished (Paindavoine et al.,

10 1986). However, it was discovered that two forms of T.b.gambiense existed (Paindavoine et

11 al., 1986; 1989) one of which was probably the classical T.b.gambiense (Type 1) and one

12 which has been called either non-gambiense or Type 2 or "Bouafle" (Gibson, 1986;

13 Paindavoine et al., 1989; Gibson, 2007). This latter group is relatively rare and may constitute

14 a variant of T.b.brucei that has acquired human infectivity (Gibson, 2007). RFLP methods

15 were developed using repetitive DNA sequences including the ribosomal RNA genes to

16 investigate RFLP variation using multiple loci (Hide et al., 1990). Although these data could

17 not be interpreted genetically and could not detect mixtures of trypanosomes, they offered a

18 highly discriminatory approach to "fingerprinting" trypanosome isolates. The RFLP data

19 combined with numerical taxonomy clearly distinguished the two types of T.b.gambiense and

20 showed that the Type 2 appeared to be a subset of West African T.b.brucei strains (Hide et

21 al., 1990). Furthermore, comparison of two T.b.rhodesiense foci in Zambia and Uganda

22 showed that there were two genetically distinct groups in the two foci (Hide et al., 1991). A

23 detailed analysis of the 1988 sleeping sickness epidemic in Tororo, Uganda, showed that the

24 human isolates (ie T.b.rhodesiense) were genetically homogeneous and could be clearly 
1 distinguished from non-human isolates (ie T.b.brucei) by numerical taxonomy (Hide et al.,

2 1994) using RFLP data. Furthermore, the T.b.rhodesiense stocks were found to be frequent

3 in cattle and were closely related to isolates from previous epidemics since the 1960s in that

4 region. Combining molecular and traditional epidemiological data it was shown that humans

5 were five times more likely to acquire sleeping sickness via cattle than from other humans

6 (Hide et al., 1996), thus demonstrating the importance of cattle as a reservoir for sleeping

7 sickness during an epidemic (Hide, 1999). Comparison of epidemic and endemic areas of

8 sleeping sickness (Hide et al., 1998) and areas free of sleeping sickness (Hide et al., 2000)

9 revealed the presence of the same human infective strains in patients, cattle and tsetse

10 suggesting that the presence of a human infective strain was not the only factor determining

11 whether an epidemic developed.

12 It was observed that T.b.rhodesiense isolates circulating in the Tororo region of Uganda were

13 genetically homogeneous whilst the sympatric T.b.brucei isolates were much more diverse

14 (Hide et al., 1994). This raised the question as to the contribution of genetic exchange in

15 "field" populations of trypanosomes. High levels of genetic exchange would facilitate the

16 "spread" of phenotypes, such as human infectivity and drug resistance, potentially into new

17 populations and could possibly explain the emergence of new epidemics. This collection of

18 stocks offered an opportunity to investigate this question since they were isolated at the

19 same place and time. However, as suitable DNA based techniques were not available, it was

20 necessary to resort to MLEE analysis to show that genetic exchange was frequent in

21 T.b.brucei but epidemic in T.b.rhodesiense (Hide et al., 1994).

22 This suggested that genetic exchange could contribute to the genetic variation in

23 trypanosome isolates in the field and importantly could contribute to the epidemiology of the

24 disease. Two problems beset further analysis of these questions. Firstly, the inability to 
1 genetically interpret multilocus RFLP markers and, secondly, despite DNA based methods

2 providing higher levels of resolution, the requirement for sufficient pure parasite material for

3 analysis.

6 The advent of PCR to amplify and identify DNA at source or from small amounts of parasite

7 material - such as a single trypanosome (Macleod et al., 1997; Cox et al., 2005) - offered tremendous advantages particularly in the area of diagnosis. In terms of epidemiological analysis, where multilocus analysis was clearly important, this was more difficult. Some single locus typing such as PCR-RFLP were found to be of limited use (Tilley \& Hide, 2001).

11 Random Amplification of Polymorphic DNA (RAPD) analysis was one of the initial solutions to PCR amplification of multiple loci (Mathieu-Daude et al., 1995; Stevens et al., 1995). This technique relied on the use of a short single PCR primer which randomly annealed to the 14 DNA sample, a banding pattern was produced for each stock and numerical taxonomy used to assess the relatedness of stocks with each other. This was a good multilocus approach, useful with small amounts of DNA, which probably sampled the full extent of the genome rather than being restricted to certain genes or parts of the genome. However, this method is highly susceptible to contaminating host or other DNA - bands will be generated from any DNA template, it is not possible to interpret genetically and is unable to detect mixed infections of trypanosomes. A similar approach, Amplified Fragment Length Polymorphism

21 (AFLP), uses PCR primers based on restriction enzyme sites to look for variation between trypanosome stocks (Agbo et al., 2002) and has the same advantages and disadvantages of RAPD analysis. To overcome the problem of contaminating host DNA, a technique termed MGE-PCR (Mobile Genetic Element PCR) was developed utilising the positional variation of 
1 mobile genetic elements to detect genetic variation between parasite isolates (Hide \& Tilley,

2 2001; Terry et al., 2001; Tilley et al., 2003). Using a single primer designed to be specific for

3 the repeat regions of the RIME mobile genetic element of T.brucei, amplification of bands

4 between adjacent RIME elements generates a banding pattern which varies from stock to

5 stock based on differences in the positions of RIME elements and so can be used to identify

6 groups of similar isolates by numerical taxonomy. This approach has the advantage that it is

7 PCR-based, specific to T.brucei DNA, multilocus, representative of a significant portion of the

8 genome and generates a single "fingerprint" for each isolate or stock. The disadvantages are

9 that it cannot detect mixed infections and it cannot be interpreted genetically.

10 The markers that offer the greatest prospects are the mini- and micro-satellite markers (eg

11 Macleod et al., 1997; Biteau et al., 2000). These short repeated sequences vary in length or

12 sequence between trypanosome strains and can be amplified and analysed by population

13 genetic methods (MacLeod et al., 2001a). Such markers can be used to detect mixed

14 infections (MacLeod et al., 1999; Koffi et al., 2007), are highly specific and sensitive tools for

15 detection of T.brucei ssp and can be used without the need to amplify parasites in rodents

16 (Morrison et al., 2007). The development of PCR based methods for detailed analysis of

17 trypanosome stocks has opened up many avenues of research. In the remainder of this

18 review we will focus on the contributions of these techniques to our understanding of the

19 diversity of trypanosome isolates in the field, the role of genetic exchange in the

20 epidemiology of sleeping sickness, the nature of human infective strains and the generation

21 of sleeping sickness epidemics.

22 Many studies have examined the diversity of T.brucei stocks obtained from field isolates.

23 What is clear is that the initial biochemical and molecular studies were correct in suggesting

24 substructuring within each of the subspecies T.b.gambiense and T.b.rhodesiense. Analyses 
1 of stocks of T.b.gambiense Type 1 clearly show this group to be genetically highly

2 homogeneous within a focus and distinguishable from T.b.brucei or T.b.rhodesiense by

3 RAPD (Jamonneau et al., 2002) microsatellite analysis (Biteau et al., 2000; Jamonneau et

4 al., 2002), minisatellite analysis (MacLeod et al., 2001a; MacLeod 2001b; MacLeod 2001c),

5 MGE-PCR (Simo et al., 2005) and AFLP (Simo et al., 2008). In most cases, Type 1

6 T.b.gambiense has been shown to be genetically homogeneous despite differing symptoms

7 in humans being associated with identical parasite genotypes (Jamonneau et al., 2002).

8 However, more detailed studies have revealed significant differences between isolates from

9 different foci within the Type 1 group (Truc et al., 2002; Morrison et al., 2008; Koffi et al.,

10 2009). Interestingly, a new group of T.b.gambiense was identified by micro- and mini-

11 satellites that was associated with asymptomatic disease in humans (Jamonneau et al.,

12 2004a). It is clear from the microsatellite studies that T.b.gambiense Type 1 is clonal and

13 very limited, if any, genetic exchange occurs in the field (Morrison et al., 2008; Koffi et al.,

14 2009).

15 The clonal nature of T.b.gambiense has allowed it to be clearly identified and shown to be

16 zoonotic. Early isoenzyme studies demonstrated its presence in dogs, pigs, bovines and wild

17 game (Mehlitz et al., 1982) suggesting a possible animal reservoir. Recent research using

18 PCR based tools have shown that pigs (Njiokou et al., 2006; Simo et al., 2006) and wild

19 fauna (Njiokou et al., 2006) have the potential to be animal reservoirs. Although in another

20 study, using microsatellite analysis, pigs were not considered to be an active reservoir

21 (Jamoneau et al., 2004b). It is also possible that untreated, parasitologically unconfirmed,

22 seropositive individuals could also act as a human reservoir (Garcia et al., 2006; Checchi et

23 al., 2008). In a clonal pathogen, such as T.b.gambiense, the lack of genetic exchange is 
1 helpful since it ensures a reasonable level of stability and reproducibility in genetic markers

2 for use in epidemiological studies.

3 As with T.b.gambiense, minisatellite analysis has been used to investigate whether

4 T.b.rhodesiense is clonal (MacLeod et al., 2000). When the sympatric stocks from Tororo,

5 Uganda (Hide et al., 1994), were examined T.b.rhodesiense was found to be clonal

6 suggesting that little or no genetic exchange is occurring in this population (MacLeod et al.,

7 2000). This is in contrast with earlier MLEE studies, using the same stocks, that suggested it

8 was epidemic (Hide et al., 1994) - that is, genetic exchange masked by the amplification of a

9 small number of genotypes. This discrepancy might be explained by the more detailed and

10 sophisticated analyses available using minisatellites.

11 However, as predicted by the earlier MLEE and RFLP studies, genetic differences between

12 different geographical populations can be clearly detected by minisatellite DNA analysis such

13 as between Uganda and Zambia (MacLeod et al., 2001b) and using polymorphism in the

14 SRA (Serum Resistant Associated) gene between populations in Uganda and Malawi or

15 Zambia (Gibson et al., 2002; McLean et al., 2004).

16 The SRA gene (De Greef et al., 1989) has been shown to be capable of transforming a non

17 human-infective T.brucei to a human-infective phenotype (Xong et al., 1998). This gene is

18 present in all human-infective field samples from East Africa and absent in animal isolates and has consequently been used as a diagnostic tool for T.b.rhodesiense (Welburn et al.,

20 2001; Gibson et al., 2002). Although, this gene looks promising as the candidate gene

21 determining human infectivity in T.brucei, it is clearly absent from both T.b.gambiense Type 1

22 and 2 (Turner et al., 2004) showing that there is more than one mechanism that determines

23 human infectivity. There is growing evidence to suggest that in at least some epidemics, such 
1 as the 1998 epidemic in Soroti in Uganda, that the spread of trypanosomes expressing the

2 SRA gene may be responsible for the generation of epidemics (Welburn et al., 2001).

4 MOLECULAR EPIDEMIOLOGY: THE ORIGINS AND DYNAMICS OF TRYPANOSOMA

5 BRUCEI RHODESIENSE SLEEPING SICKNESS EPIDEMICS

6 The 1988 Tororo sleeping sickness epidemic in Uganda resulted in a legacy of trypanosome

7 stocks isolated during an epidemic (Hide et al., 1994). In addition to analysis by RFLP, they

8 have been analysed using minisatellite markers (MacLeod et al., 2000), MGE-PCR (Tilley et

9 al., 2003) and the SRA gene (Welburn et al., 2001). A clear distinction has emerged between the human infective stocks and non human-infective stocks in this region. The finding that

11 cattle are an important reservoir for human infective stocks (Hide et al., 1996) has been confirmed by more recent studies (Welburn et al., 2001). Isolates collected from humans ten

13 years on from this epidemic have been shown by MGE-PCR to contain some identical 14 genotypes thus confirming the stability of genotypes within this focus (Tilley et al., 2003). A new epidemic started in December 1998 in Soroti, Uganda (Fevre et al., 2001), and is currently ongoing and spreading north and west (Fevre et al., 2005). Based on the evidence that cattle are an important reservoir for sleeping sickness, it has been postulated that cattle infected with human-infective T.brucei were imported from the Tororo District into Soroti

19 (Fevre et al., 2001). These cattle were then fed upon by local tsetse, passed onto the local people and the epidemic started. Statistical analysis of the location of sleeping sickness cases in relation to the Soroti cattle market (Fevre et al., 2001) and the presence of SRA positive T.brucei isolated from the cattle at this market has supported this view (Welburn et al., 2001). Other studies conducted on T.brucei isolates collected from Soroti suggest that they have a different clinical disease profile (eg more chronic presentation) to isolates 
1 collected in Tororo and also the corresponding microsatellite profiles were found to be

2 different (McLean et al., 2007). This supports the view that, although some of the isolates

3 have probably originated in Tororo, there may be other origins of these T.brucei isolates.

4 These results clearly show that the importation of cattle from a high risk area may have been

5 responsible for the generation of this new epidemic. It also shows the importance of tracking

6 cattle movements particularly from high risk areas, such as Tororo, and the identification of

7 the geographical locations of high risk areas.

8 Based on the extensive molecular epidemiological data from Uganda, it is possible to

9 formulate a model of how sleeping sickness foci are generated and how epidemics develop.

10 It is clear from the data that foci, such as the Busoga focus, must have the presence of a

11 human infective T.brucei strain (“T.b.rhodesiense"). Molecular epidemiological studies

12 suggest that this may be a single predominant clonal strain which remains stable over time

13 (although multiple clonal strains could also be present). New epidemics can be generated by

14 the influx of a human strain into an area previously occupied by only animal strains. This

15 could be by the importation of a reservoir species, such as cattle, infected with a human

16 strain. Local socio-political customs or events may determine the amplitude and duration of

17 the epidemic. Despite having a wide antigenic repertoire, clonality will ensure that any given

18 T.b.rhodesiense strain will have reduced genetic variability. Consequently, with time, a local

19 human population will become immune/less susceptible or subjected to health interventions

20 causing the epidemic to decline. On entering a naïve population, such as Soroti, clonal

21 human strains would spread quickly. It is clear that different foci appear to have genetically

22 different human strains (T.b.rhodesiense or T.b.gambiense) which could be generated either

23 by the SRA gene being introduced into a new genetic background or by the introduction of

24 one of the other mechanisms of human infectivity emerging in a previously non human- 
1 infective strain. This mechanism could involve genetic exchange between a non human- and

2 a human-infective strain. With time and selection through humans, emergent human strains

3 might become established and generate new foci. The data suggest that in the field genetic

4 exchange occurs but is masked by the epidemic spread of a small number of strains. The

5 relatively small numbers of human infective strains, the relatively small number of foci and

6 the long term stability of foci might suggest that there is a minimal effect of genetic exchange

7 in generating new human strains and foci. However, in practice, the generation of even a

8 single new focus creates a significant health issue. Important questions arise from this model.

9 Are there low frequencies of genetic exchange generating new human-infective strains or is the generation of new human-infective strains by genetic exchange frequent but fixation of

11 those strains within the population (to produce a focus) infrequent? Also, given the relatively

12 limited data from foci in Zambia, Tanzania, Malawi and Mozambique, are the conclusions

13 from the Ugandan studies typical for T.b.rhodesiense? As can be seen from this review, the

14 molecular tools are now available to address such questions.

15 As with any model, this one generates more questions than it answers. We have come along 16 way to understanding the generation of sleeping sickness epidemics but further questions 17 need to be addressed. The future of molecular epidemiology will be based on our increasing 18 ability to be able to conduct large scale epidemiological studies which will involve carefully controlled sampling strategies, application of high throughput whole genome technologies for both host and parasite, development of Single Nucleotide Polymorphism (SNP) chips,

21 development of bioinformatics tools and integration with mathematical models of disease transmission. These approaches and technologies provide opportunities for the future and offer the prospect of addressing some of the questions raised in this review. 


\section{ACKNOWLEDGEMENTS}

2 We would like to acknowledge all of our colleagues who have contributed to the studies we

3 have reviewed here. While we have tried to provide appropriate coverage in this review, we

4 acknowledge that a great deal of important work could not be included here for reasons of 5 space. 
2

3

4

7 BITEAU, N., BRINGAUD, F., GIBSON, W.C., TRUC, P. and BALTZ, T. (2000)

\section{REFERENCES}

AGBO, E.E.C., MAJIWA, P.A.O., CLAASSEN, H.J. and TE PAS, M.F.W. (2002) Molecular variation of Trypanosoma brucei subspecies as revealed by AFLP fingerprinting. Parasitology 124, 249-358.

Characterization of Trypanozoon isolates using a repeated coding sequence and microsatellite markers

Molecular and Biochemical Parasitology 105,185-201.

2 BORST, P., FASE-FOWLER, F., and GIBSON W.C. (1981). Quantitation of genetic differences between Trypanosoma brucei gambiense, rhodesiense and brucei by restriction enzyme analysis of kinetoplast DNA. Molecular and Biochemical Parasitology 3:117-131. .

CARTER, R. and WALLIKER, D. (1975). New observations on the malaria parasites of rodents of the Central African Republic - Plasmodium vinckei petteri subsp. nov. and Plasmodium chabaudi Landau, 1965. Annals of Tropical Medicine and Parasitology. 69,18796.

CHECCHI, F., FILIPE, J.A., BARRETT, M.P. and CHANDRAMOHAN, D. (2008). The natural progression of gambiense sleeping sickness: what is the evidence? PLoS Neglected Tropical Diseases. 2303. 
1 COX, A., TILLEY, A., MCODIMBA, F., FYFE, J., HIDE, G. and WELBURN, S.C. (2005). A

2 PCR based assay for detection and differentiation of African trypanosome species in blood.

3 Experimental Parasitology, 111, 24-29.

4

5 DE GREEF, C., IMBERECHTS, H., MATTHYSSENS, G., VAN MEIRVENNE, N., and

6 HAMERS, R. (1989). A gene expressed only in serum-resistant variants of Trypanosoma

7 brucei rhodesiense. Molecular and Biochemical Parasitology, 36,169-176.

9 FÈVRE, E.M., COLEMAN, P.G., ODIIT, M.D., MAGONA, J., WELBURN, S.C. and WOOLHOUSE, M.E.J. (2001). The origins of a new sleeping sickness outbreak (caused by

11 Trypanosoma brucei infection) in eastern Uganda. The Lancet 358, 625-628.

FÈVRE, E.M., PICOZZI, K., FYFE, J., WAISWA, C., ODIIT, M., COLEMAN, P.G. and WELBURN S.C. (2005). A burgeoning epidemic of sleeping sickness in Uganda. The Lancet. 366, 745-747.

GARCIA, A., COURTIN, D., SOLANO, P., KOFFI, M. and JAMONNEAU, V. (2006). Human African trypanosomiasis: connecting parasite and host genetics. Trends in Parasitology 22, 405-409.

21 GIBSON, W.C. (1986). Will the real Trypansoma brucei gambiense please stand up? 22 Parasitology Today 2, 255-257. 
1 GIBSON, W.C. (1989). Analysis of a genetic cross between Trypanosoma brucei

2 rhodesiense and T. b. brucei. Parasitology, 99, 391-402.

4 GIBSON, W.C. (2009) Species-specific probes for the identification of the African tsetse-

5 transmitted trypanosomes. Parasitology. In Press (current volume).

7 GIBSON, W., MEHLITZ, D., LANHAM, S.M. and GODFREY, D,G. (1978). The identification

8 of Trypanosoma brucei gambiense in Liberian pigs and dogs by isoenzymes and by

9 resistance to human plasma. Tropenmedezin und Parasitologie, 29, 335-45

11 GIBSON, W.C., MARSHALL, T.F. DE C. and GODFREY, D.G. (1980) Numerical analysis of enzyme polymorphism. A new approach to the epidemiology and taxonomy of trypanosomes 13 of the genus Trypanozoon. Advances in Parasitology 18, 175-245.

GIBSON, W., BORST, P. and FASE-FOWLER, F. (1985). Further analysis of intraspecific 16 variation in Trypanosoma brucei using restriction site polymorphisms in the maxi-circle of 17 kinetoplast DNA. Molecular and Biochemical Parasitology, 15, 21-36

GIBSON, W.C., BACKHOUSE, T. and GRIFFITHS, A. (2002) The human serum resistance associated gene is ubiquitous and conserved in Trypanosoma brucei rhodesiense throughout

21 East Africa. Infection, Genetics and Evolution 1, 207-214.

23 GIBSON, W.C. (2007) Resolution of the species problem in African trypanosomes.

24 International Journal for Parasitology, 37, 829 - 838. 
1 GODFREY, D.G. (1978). Identification of economically important parasites. Nature, 273, 600-

2604.

3

4 HIDE, G. (1999) The history of sleeping sickness in East Africa. Clinical Microbiological

5 Reviews 12, 112-125.

6

7 HIDE, G., CATTAND, P., LE RAY, D., BARRY, J.D. and TAIT, A. (1990). The identification of

8 T. brucei subspecies using repetitive DNA sequences. Molecular and Biochemical 9 Parasitology, 39, 213-226.

11 HIDE, G. and TAIT, A. (1991) The molecular epidemiology of parasites. Experientia 47, 128142.

13

14 HIDE, G., BUCHANAN, N., WELBURN, S.C., MAUDLIN, I., BARRY, J.D. and TAIT, A. (1991). Trypanosoma brucei rhodesiense: Characterization of stocks from Zambia, Kenya 16 and Uganda using repetitive DNA probes. Experimental Parasitology, 72, 430-439.

HIDE, G., WELBURN, S.C., TAIT, A. and MAUDLIN, I. (1994). Epidemiological relationships of Trypanosoma brucei stocks from South East Uganda: Evidence for different population structures in human and non human trypanosomes. Parasitology,109, 95-111.

21

22 HIDE, G., TAIT, A., MAUDLIN, I. and WELBURN, S.C. (1996). The origins, dynamics and 23 generation of Trypanosoma brucei rhodesiense epidemics in East Africa. Parasitology Today, $24 \quad 12,50-55$. 
2 HIDE G., ANGUS, S., HOLMES, P.H., MAUDLIN, I. and WELBURN, S.C. (1998).

3 Comparison of Trypanosoma brucei strains circulating in an endemic and an epidemic

4 sleeping sickness focus. Experimental Parasitology. 89, 21-29.

5

6 HIDE, G., TILLEY, A., WELBURN, S., MAUDLIN, I. and TAIT, A. (2000). Trypanosoma

7 brucei: identification of trypanosomes with genotypic similarity to human infective isolates in

8 tsetse isolated from a region free of human sleeping sickness. Experimental Parasitology. 96,

$9 \quad 67-74$.

10

11 HIDE, G. and TILLEY, A. (2001) Use of mobile genetic elements as tools for molecular

12 epidemiology. International Journal of Parasitology, 31, 599-602.

13

14 JamonneaU, V., Garcia, A., RAVEl, S., CUNY, G., OURY, B., SOlano, P., 15 N'GUeSSAN, P., N'DRI, L., SANON, R., FRÉZIL, J.L. and TRUC, P. (2002). Genetic 16 characterization of Trypanosoma brucei gambiense and clinical evolution of human African 17 trypanosomiasis in Côte d'Ivoire. Tropical Medicine and International Health, 7, 610-621.

JAMONNeAU, V., RAVEL, S., GARCIA, A., KOFFI, M., TRUC, P., LAVEISSIÈRE, C., HERDER, S., GRÉBAUt, P., CUNY, G. and SOlANO, P. (2004a). Characterization of

21 Trypanosoma brucei s.l. infecting asymptomatic sleeping-sickness patients in Côte d'Ivoire: a 22 new genetic group? Annals of Tropical Medicine and Parasitology, 98, 329-37. 
1 JAMONNEAU, V., RAVEL, S., KOFFI, M., KABA, D., ZEZE, D.G., NDRI, L., SANE, B.,

2 COULIBALY, B., CUNY, G. and SOLANO, P, (2004b). Mixed infections of trypanosomes in

3 tsetse and pigs and their epidemiological significance in a sleeping sickness focus of Côte 4 d'Ivoire. Parasitology. 129, 693-702.

6 JENNI, L., MARTI, S., SCHWEIZER, J., BETSCHART, B., LE PAGE, R.W.F., WELLS, J.M.,

7 TAIT, A., PAINDAVOINE, P., PAYS, E. and STEINERT, M. (1986). Hybrid formation between 8 African trypanosomes during cyclical transmission. Nature 322, 173-175.

KILGOUR, V. and GODFREY, D.G. (1973). Isoenzymes of alanine aminotransferase as 11 possible specific characters of trypanosomes. Transactions of the Royal Society of Tropical 12 Medicine and Hygiene. 67, 11.

KOFFI, M., SOLANO, P., BARNABÉ, C., DE MEEÛS, T., BUCHETON, B., CUNY. G. and JAMONNEAU, V. (2007). Genetic characterisation of Trypanosoma brucei s.l. using microsatellite typing: new perspectives for the molecular epidemiology of human African 17 trypanosomiasis. Infection Genetics and Evolution. 7, 675-84.

KOFFI. M., DE MEEÛS, T., BUCHETON, B., SOlANO, P., CAMARA, M., KABA, D., CUNY, gambiense, the agent of sleeping sickness in Western Africa. Proceedings of the National Academy of Sciences, USA. 106, 209-214. 
1 MACLEAN, L., CHISI, J.E., ODIIT, M., GIBSON, W.C., FERRIS, V., PICOZZI, K. and

2 STERNBERG, J.M. (2004). Severity of human african trypanosomiasis in East Africa is

3 associated with geographic location, parasite genotype, and host inflammatory cytokine 4 response profile. Infection and Immunity, 72, 7040-7044.

6 MACLEAN, L., ODIIT, M., MACLEOD, A., MORRISON, L., SWEENEY, L., COOPER, A.,

7 KENNEDY, P.G. and STERNBERG, J.M. (2007). Spatially and genetically distinct African

8 trypanosome virulence variants defined by host interferon-gamma response. Journal of 9 Infectious Diseases, 196,1620-1628.

MACLEOD, A., TURNER, C.M.R., and TAIT, A. (1997). Detection of single copy gene sequences from single trypanosomes. Molecular and Biochemical Parasitology, 84, 267-270.

MACLEOD, A., TURNER, C.M.R. and TAIT, A. (1999). A high level of mixed Trypanosoma

brucei infections in tsetse flies detected by three hypervariable minisatellites. Molecular and 16 Biochemical Parasitology, 102, 237-248.

MACLEOD, A., TWEEDIE, A., WELBURN, S.C., MAUDLIN, I., TURNER, M.J. and TAIT, A.

(2000). Minisatellite marker analysis of Trypanosoma brucei: reconciliation of clonal, panmictic and epidemic population genetic theories. Proceedings of the National Academy of

21 Sciences $97,13442-13447$ 
1 MACLEOD, A., TURNER, C.M.R. and TAIT, A. (2001a). The detection of geographical

2 substructuring of Trypanosoma brucei populations by the analysis of minisatellite

3 polymorphisms. Parasitology 123, 475-482.

4

5 MACLEOD, A., TAIT, A. and TURNER, C.M. (2001b). The population genetics of

6 Trypanosoma brucei and the origin of human infectivity. Philosophical Transactions of the

7 Royal Society of London B, 356, 1035-1044.

9 MACLEOD, A., WELBURN, S., MAUDLIN, I., TURNER, C.M. and TAIT, A. (2001c). Evidence for multiple origins of human infectivity in Trypanosoma brucei revealed by minisatellite 11 variant repeat mapping. Journal of Molecular Evolution 52, 290-301.

MATHIEU-DAUDÉ, F. and TIBAYRENC, M. (1994). Isozyme variability of Trypanosoma 14 brucei s.I.: genetic, taxonomic, and epidemiological significance. Experimental Parasitology, $15 \quad 78,1-19$.

MATHIEU-DAUDE, F., STEVENS, J., WELSH, J., TIBAYRENC, M. and MCLELLAND, M. (1995). Genetic diversity and population structure of Trypanosoma brucei: clonality versus sexuality. Molecular and Biochemical Parasitology 72, 89-101.

MAYNARD SMITH, J., SMITH, N.H., O'ROURKE, M. and SPRATT, B.G. (1993). How clonal are bacteria? Proceedings of the National Academy of Sciences 90, 4384-4388. 
1 MEHLITZ, D., ZILLMANN, U., SCOTT, C.M., AND GODFREY, D.G.(1982). Epidemiological

2 studies on the animal reservoir of Gambiense sleeping sickness. Part III. Characterization of

3 trypanozoon stocks by isoenzymes and sensitivity to human serum. Tropenmedezin und

4 Parasitologie, 33, 113-118.

5

6 MILES, M.A., TOYE, P.J., OSWALD, SC. and GODFREY, D.G. (1977). The identification by

7 isoenzyme patterns of two distinct strain-groups of Trypanosoma cruzi, circulating

8 independently in a rural area of Brazil. Transactions of the Royal Society of Tropical Medicine

9 and Hygiene. 71, 217-225.

MORRISON, L.J., MCCORMACK, G., SWEENEY, L., LIKEUFACK, A.C., TRUC, P.,

12 TURNER, C.M., TAIT, A. and MACLEOD, A. (2007). Use of multiple displacement

13 amplification to increase the detection and genotyping of trypanosoma species samples

14 immobilized on FTA filters. American Journal of Tropical Medicine and Hygiene, 76,1132-

151137.

16

MORRISON, L.J., TAIT, A., MCCORMACK, G., SWEENEY, L., BLACK, A., TRUC, P., LIKEUFACK, A.C., TURNER, C.M. and MACLEOD, A. (2008). Trypanosoma brucei gambiense Type 1 populations from human patients are clonal and display geographical genetic differentiation. Infection Genetics and Evolution, 8, 847-54.

NJIOKOU, F., LAVEISSIÈRE, C., SIMO, G., NKININ, S., GRÉBAUT, P., CUNY, G. and 23 HERDER, S. (2006). Wild fauna as a probable animal reservoir for Trypanosoma brucei 24 gambiense in Cameroon. Infection Genetics and Evolution, 6, 147-53. 
2 PAINDAVOINE, P., PAYS, E., LAURENT, M., GELTMEYER, Y., LE RAY, D., MEHLITZ, D.

3 and STEINERT, M. (1986). The use of DNA hybridization and numerical taxonomy in

4 determining relationships between Trypanosoma brucei stocks and subspecies. Parasitology.

$592,31-50$.

7 PAINDAVOINE, P., ZAMPETTI-BOSSELER, F., COQUELET, H., PAYS, E. and STEINERT,

8 M. (1989). Different allele frequencies in Trypanosoma brucei brucei and Trypanosoma

9 brucei gambiense populations. Molecular and Biochemical Parasitology, 32, 61-71.

11 SIMO, G., HERDER, S., NJIOKOU, F., ASONGANYI, T., TILLEY, A. and CUNY, G. (2005).

12 Trypanosoma brucei s.I.: characterisation of stocks from Central Africa by PCR analysis of

13 mobile genetic elements. Experimental Parasitology, 110, 353-62.

14

SIMO, G., ASONGANYI, T., NKININ, S.W., NJIOKOU, F. and HERDER, S. (2006). High 16 prevalence of Trypanosoma brucei gambiense group 1 in pigs from the Fontem sleeping 17 sickness focus in Cameroon. Veterinary Parasitology, 139, 57-66.

SIMO, G., CUNY, G., DEMONCHY, R. AND HERDER, S. (2008). Trypanosoma brucei gambiense: study of population genetic structure of Central African stocks using amplified

21 fragment length polymorphism (AFLP). Experimental Parasitology, 118, 172-80. 
1 STEVENS, J.R. and TIBAYRENC, M. (1995). Detection of linkage disequilibrium in

2 Trypanosoma brucei isolated from tsetse flies and characterized by RAPD analysis and 3 isoenzymes. Parasitology, 110, 181-186.

4

5 TAIT, A. (1970a). Genetics of NADP isocitrate dehydrogenase in Paramecium aurelia.

$6 \quad$ Nature, 225, 181-182.

7

8 TAIT, A. (1970b). Enzyme variation between syngens in Paramecium aurelia. Biochemical 9 Genetics, 4, 461-70.

10

11 TAIT, A. (1980) Evidence for diploidy and mating in trypanosomes. Nature 287, 536-538.

12

13 TAIT, A., BABIKER, E.A. and LE RAY, D. (1984). Enzyme variation in Trypanosoma brucei 14 ssp. I. Evidence for the subspeciation of Trypanosoma brucei gambiense. Parasitology 89, $15 \quad 311-326$.

16

17 TAIT, A., BARRY, J.D., WINK, R., SANDERSON, A. and CROWE, J.S. (1985). Enzyme 18 variation in Trypanosoma brucei ssp. II. Evidence for $T$. $b$. rhodesiense being a subset of 19 variants of T. b. brucei. Parasitology. 90, 89-100.

20

21 TAIT, A. and TURNER, C.M.R. (1990). Genetic exchange in Trypanosoma brucei. 22 Parasitology Today 6, 70-75. 
1 TERRY R.S, SMITH, J.E., DUNCANSON, P. and HIDE, G. (2001). MGE-PCR: a novel

2 approach to the analysis of Toxoplasma gondii strain differentiation using mobile genetic

3 elements. International Journal of Parasitology, 31, 155-161.

4

5 TIBAYRENC, M., KJELLBERG, F. and AYALA, F.J. (1990). A clonal theory of parasitic 6 protozoa: the population structures of Entamoeba, Giardia, Leishmania, Naegleria, 7 Plasmodium, Trichomonas and Trypanosoma and their medical and taxonomic consequences. Proceedings of the National Academy of Sciences, 87, 2414-2418.

TILLEY, A. and HIDE, G. (2001). Characterization of Trypanosoma brucei stocks by 11 Intergenic Region Typing. Annals of Tropical Medicine and Parasitology, 95, 617-621.

TILLEY, A, WELBURN, S.C., FEVRE, E., FEIL, E.J. and HIDE, G. (2003). Trypanosoma 14 brucei: Trypanosome strain typing using PCR analysis of mobile genetic elements (MGE15 PCR). Experimental Parasitology, 104, 26 -32.

17 TRUC, P., AERTS, D., MCNAMARA, J. J., CLAES, Y., ALLINGHAM, R. LE RAY, D. and 18 GODFREY, D. G. (1992). Direct isolation in vitro of Typanosoma brucei from man and other animals, and its potential value for the diagnosis of gambian trypanosomiasis. Transactions 20 of the Royol Society of Tropical Medicine and Hygiene. 86, 627-629.

22 TRUC, P., RAVEL, S., JAMONNEAU, V., N'GUESSAN, P. AND CUNY, G. (2002). Genetic 23 variability within Trypanosoma brucei gambiense: evidence for the circulation of different 
1 genotypes in human African trypanosomiasis patients in Côte d'Ivoire. Transactions of the

2 Royal Society of Tropical Medicine and Hygiene, 96, 52-55.

4 TURNER, C.M., MCLELLAN, S., LINDERGARD, L.A., BISONI, L., TAIT, A. and MACLEOD

5 A. (2004). Human infectivity trait in Trypanosoma brucei: stability, heritability and relationship 6 to SRA expression. Parasitology. 129, 445-454

7

8 WALLIKER, D., CARTER, R. and MORGAN, S. (1971). Genetic recombination in malaria 9 parasites. Nature, 232, 561-562.

WALLIKER, D., CARTER, R. and MORGAN, S. (1973). Genetic recombination in

Plasmodium berghei. Parasitology. 66, 309-320.

WELBURN, S.C., PICOZZI, K., FÈVRE, E.M., COLEMAN, P.G., ODIIT, M., CARRINGTON,

M. and MAUDLIN, I. (2001). Identification of human infective trypanosomes in animal

reservoirs of sleeping sickness in Uganda by means of serum-resistance-associated (SRA)

17 gene. The Lancet, 358, 2017-2019.

XONG, H.V., VANHAMME, L., CHAMEKH, M., CHIMFWEMBE, C.E., VAN DEN ABBEELE,

J., PAYS, A., VAN MEIRVENNE, N., HAMERS, R., DE BAETSELIER, P. and PAYS, E.

(1998). A VSG expression site-associated gene confers resistance to human serum in

Trypanosoma rhodesiense. Cell., 95, 839-346. 
TABLE LEGENDS

2

3 Table 1. Characteristics of the subspecies of Trypanosoma brucei

4

5 Table 2 Molecular epidemiological methods for analysing African trypanosomes. The table

6 shows some of the characteristics of each method: 1. Subspecies discrimination, ability to

7 discriminate between the three subspecies of T.brucei. 2. Identification of populations or

8 groups, the ability to define populations or groups within the subspecies. 3. Population

9 genetics, the ability to be interpreted genetically to enable population genetics to be carried

out. 4. Detection of mixed infections, able to detect mixed infections or not to be confounded

by the presence of mixed infections. 5. PCR based amplification of DNA, can be used directly

12 on blood or tissue samples without the need to amplify parasites in experimental animals or

13 in vitro. 6. Requires pure parasites, parasites need to be amplified and purified. MLEE,

14 Multilocus Enzyme Electrophoresis; RFLP, Restriction Fragment Length Polymorphism;

15 PCR-RFLP, PCR amplified RFLP; RAPD, Randomly Amplified Polymorphic DNA; AFLP, 16 Amplified Fragment Length Polymorphism; MGE-PCR, Mobile Genetic Element - PCR;

17 Minisatellites and Microsatellites, analysis of length polymorphism in highly repeated

18 sequences; SRA, detection of the Serum Resistance Associated gene which is found in human infective T.b.rhodesiense. 


\section{FIGURE LEGEND}

2 Figure 1 A map of the Busoga focus of T.b.rhodesiense sleeping sickness in Uganda. Recent 3 epidemics occurred in Tororo (1988 to date) and Soroti (1998 to date). 